\title{
ERRATA : CORRUZIONE E VIOLENZASISTEMICHE: RIFLETTENDO SUL RAPPORTO TRA POLITICA E DIRITTO A PARTIRE DA "TANGENTOPOLI"
}

\author{
ERRATA: SYSTEM CORRUPTION AND SYSTEMIC VIOLENCE BETWEEN LAW \\ AND POLITICS: REFLECTIONS FROM THE ITALIAN "TANGENTOPOLI"
}

\section{ERRATA CORRUPÇÃO E VIOLENCIA SISTÊMICAS: REFLEXÕES A PARTIR DE "TANGENTOPOLI"}

Sandra Regina Martini ${ }^{1}$ Matteo Finco ${ }^{2}$

O artigo original foi publicado em 25 de março de 2020. https://doi.org/10.5216/rfd.v43.60177

Errata: No artigo Corruzione e violenza sistemiche: riflettendo sul rapporto tra politica e diritto a partire da "tangentopoli", com número DOI: doi.org/10.5216/rfd.v43.60177, publicado no periódico "Revista da Faculdade de Direito da UFG, v.43: p.01-16, na página 01:

Em reparo à versão original, aponta-se a inclusão da coautoria de Matteo Finco.

A versão em pdf foi modificada.

\footnotetext{
1 Possui graduação em Ciências Sociais pela Universidade do Vale do Rio dos Sinos (1983), mestrado em Educação pela Pontifícia Universidade Católica do Rio Grande do Sul (1997), doutorado em Evoluzione dei Sistemi Giuridici e Nuovi Diritti pela Università Degli Studi di Lecce (2001), Pós-doutorado em Direito (Roma Tre, 2006) e Pós-doutorado em Políticas Públicas (Universidade de Salerno, 2010). Foi Professora titular da Universidade do Vale do Rio dos Sinos, da Scuola Dottorale Internazionale Tullio Ascarelli e professora visitante da Universita Degli Studi Di Salerno. Foi diretora da Escola de Saúde Pública do Rio Grande do Sul (janeiro de 2007 a fevereiro de 2011), foi membro( de janeiro de 2008 a dezembro de 2013) do Conselho Superior da Fundação de Amparo à Pesquisa do Estado do Rio Grande do Sul (FAPERGS). Atualmente é Pesquisadora Produtividade $2 \mathrm{CNPq}$, Coordenadora do Mestrado em Direitos Humanos e pro- fessora do Centro Universitário Ritter dos Reis (Uniritter), professora-visitante no programa de pósgrad- uação em Direito da UFRGS (PPGD). É avaliadora do Basis do Ministério da Educação e Cultura e do Basis do Instituto Nacional de Estudos e Pesquisas Educacionais Anísio Teixeira. Parecerista ad hoc CNPq e CAPES. Conferencista no Brasil e no exterior. Membro da Rede Iberoamericana de Direito Sanitário. E-mail: srmartini@terra.com.br

2 Possui graduação em Scienze della Comunicazione (2008) e em Sociologia della Multiculturalità Università degli Studi di Urbino (2013), e doutorado em Social Sciences - System theory and sociology of normative and cultural processes - Università degli Studi di Macerata (2017). Jornalista (registro de jornalistas profissionais italianos). Atualmente, é bolsista de pesquisa de pós-doutorado CAPES no Centro Universitário Ritter dos Reis. E-mail: fincomatteo@gmail.com
} 


\begin{abstract}
Questo lavoro, a partire dalla teoria dei sistemi sociali di N. Luhmann, espone i concetti di "corruzione sistemica" e "violenza sistemica", analizzando l'impatto dei fenomeni che essi descrivono sui sottosistemi societari del diritto e della politica. Viene studiato un caso concreto, cioè quanto avvenuto in Italia nei primi anni '90 (il sistema corruttivo denominato "Tangentopoli", svelato grazie alle indagini di "Mani Pulite"), per identificarne le principali ripercussioni sui diritti umani e i derivanti conflitti tra media, opinione pubblica, potere giudiziario e politico. Attraverso la ricerca bibliografica e il metodo ipotetico- deduttivo, la corruzione viene concepita non soltanto come un crimine, ma come un fenomeno sociale che coinvolge la società nel suo complesso e i cittadini, intesi come individui chiamati a scegliere e ad agire responsabilmente. Proprio in virtù del riferimento ai livelli individuale e interpersonale della vita sociale, le considerazioni fatte possono applicarsi anche a contesti differenti da quello qui analizzato (Italia).
\end{abstract}

Parole chiave: Corruzione; Corruzione sistemica; Violenza sistemica; Diritti umani; Tangentopoli.

\begin{abstract}
The article adopts as framework the Social System Theory by N. Luhmann and provides an overview of the concepts of "systemic corruption" and "systemic violence", analysing how the phenomena they describe affect the subsystems of Law and Politics. The analysis of "Tangentopoli" (Italy), connected to the criminal investigation "Mani Pulite", attempts to concretely identify the most important repercussions for Human Rights and the conflicts between media, public opinion, judicial and political power. Through bibliographical and documentary research and the hypothetic-deductive method, corruption is conceived not only as a crime, but as a social phenomenon that involves the overall society as well as the citizens, conceived as individuals who are called to choose and act in a responsible way. Precisely because of this reference to the individual and interpersonal levels of social life, the considerations made may also apply to contexts different from the one analyzed here (Italy).
\end{abstract}

Keywords: Corruption; Systemic Corruption; Systemic Violence; Human Rights; Tangentopoli.

Resumo: O artigo adota a Teória dos Sistemas Sociais do sociólogo N. Luhmann e fornece um enquadramento dos conceitos de "corrupção sistêmica" e "violência sistêmica", analisando como os fenômenos que eles descrevem afetam os subsistemas do direito e da política. A análise dos eventos de "Tangentopoli" (Itália), conectados à investigação criminal "Mani Pulite", tenta identificar concretamente as repercussões no âmbito dos direitos humanos e dos conflitos entre mídia, opinião pública, poderes judiciário e político. Através de pesquisa bibliográfica e documental e do método hipotético-dedutivo, a corrupção é entendida não somente como crime, mas como fenômeno social abrangente, que envolve a sociedade como um todo bem como os cidadãos, entendidos como individuos que no dia dia são chamados para escolhas e açoes responsáveis. Precisamente por esta referência aos níveis individual e interpessoal da vida social, as considerações feitas também podem se aplicar a contextos diferentes do aqui analisado (Itália).

Palavras-chaves: Corrupção; Corrupção sistêmica; Violência sistêmica; Direitos Humanos; Tangentopoli.

\title{
INTRODUZIONE
}

Le riflessioni che seguono ${ }^{1}$, di stampo sociogiuridico, si pongono i seguenti obiettivi: fornire un inquadramento teorico - a partire dalla teoria dei sistemi sociali ${ }^{2}$ elaborata nella seconda metà del XX seculo dal sociologo tedesco Niklas Luhmann - di due concetti: "corruzione sistemica" e "violenza sistemica"; analizzare come queste influenzano due ambiti della società contemporanea (sottosistemi sociali): diritto e politica; analizzando un caso concreto di "corruzione sistemica" fra diritto e politica -l'epoca (nell'Italia dei primi anni '90) di Tangentopoli, risultato di un insieme di indagini e processi de- nominato "Mani Pulite" - identificare le principali ripercussioni nell'ambito dei diritti umani (vi- olazioni) e dei conflitti fra mass-media, potere giudiziario, potere politico, opinione pubblica. 
Si tratta di un lavoro di carattere "esplorativo", che intende stabilire le fondamenta teoriche di un progetto più ampio e che, attraverso una ricerca bibliografica e del metodo ipotetico-deduttivo, tenta di concepire la corruzione al di là dell'ambito giuridico-penale, ossia, come fenomeno sociale di ampia portata, con conseguenze nei vari livelli della vita sociale.

Un approccio teorico tanto rigoroso - la SST - si rivela essenziale per l'analisi del fenomeno della corruzione a partire da una prospettiva più ampia rispetto a quella consentita se si intende il termine nel suo senso comume. Allo stesso tempo, l'analisi sociologica viene accompagnata da una serie di riferimenti (inclusi per lo più in nota) a un testo dell'ex magistrato italiano Gherardo Colombo: nonostante il tenore totalmente diverso rispetto alla TSS, tali considerazioni indicano in maniera diretta alcuni essenziali snodi problematici della relazione fra politica, giustizia e democrazia $^{3}$ da un lato e corruzione dall'altro ${ }^{4}$. Per questa ragione, esse sono utili per portare la nostra riflessione dal piano dei processi sociali più generali della societò moderna a quello - aparentemente - più "concreto" della vita quotidiana.

\section{LA TEORIA DEI SISTEMISOCIALI}

Dal punto di vista della teoria dei sistemi sociali, la società moderna è caratterizzata da differenti sistemi di funzione, ovvero ambiti/settori chiamati a risolvere un problema specifico della società. Per realizzare tale funzione, essi operano per mezzo di codici, ovvero dei valori che permettono al sistema di ridurre la complessità, collegando le operazioni ai problemi da risolvere e permettendo, allo stesso tempo, la continuità delle operazioni stesse (comunicazione). È importante sottolineare che ogni operazione risulta necessaria per la riproduzione del sistema stesso, che in questo modo continua a esistere. Ad esempio: l'economia serve a gestire il problema dell'accesso a risorse scarse (non tutti possono possedere tutto contemporaneamente) e funziona in base al codice avere/non avere; la scienza produce sapere e il suo codice è vero/non vero; la medicina è votata alla cura delle malattie, e il codice è malato/sano. Ciò vale per anche per gli altri sistemi, come la religione, l'arte, le famiglie e i rapporti intimi e l'educazione, eccetera.

Occorre però aggiungere che la teoria di Luhmann non è una teoria critica nel senso tradizionale del termine ${ }^{5}$, ovvero essa non ha in primo luogo la pretesa di come "dovrebbero andare le cose". Al contrario, essa è una teoria descrittiva: dire che ci sono differenti sistemi che si occupano di problemi differenti non è un dogma, ma un maniera di intendere e di spiegare la realtà. Si tratta dunque di osservare e descrivere la realtà. Pertanto, non si deve ragionare in termini di "giusto" o "sbagliato" in senso morale (giudicare i sistemi), o pensare a "bene" e "male". Ogni sistema funziona secondo una logica specifica (espressa dal suo codice), che non serve a nulla valutare secondo parametri etici/morali ${ }^{6}$. D'altra parte i sistemi non sono entità impersonali che funzionano automaticamente: è ovvio che gli esseri umani (quale che sia la forma in cui è possibile concepirli: persone, individui, soggetti, corpi, coscienze) operano "all'interno" dei sistemi, e quindi sono i loro comportamenti concreti a determinare il sistema e se esso "fa bene il suo lavoro".

Cosa succede se un sistema non funziona come dovrebbe (nel senso della TSS), se non svolge la sua funzione, ovvero se opera con obbiettivi diversi dai suoi specifici, se rinuncia al proprio codice? In questo caso si parla di dedifferenziazione, ovvero il contrario della differenziazione e cioè 
una "mescolanza" tra sistemi. Ad esempio: quando un giudice non emette una sentenza fondata sulle norme di legge, ma basandosi sulle proprie idee o convinzioni morali; o quando un professore assegna un voto o un giudizio sulla base della simpatia che prova per i suoi studenti ${ }^{7}$. In questo lavoro, il nostro interesse riguarda principalmente l'analisi di due sistemi: quello del diritto e quello della politica. L'obiettivo - ripetiamo - è di avanzare delle considerazioni relative a come questi due sistemi "abbiano funzionato" e siano stati influenzati in Italia nell'epoca di Tangentopoli/Mani pulite (essenzialmente, negli anni dal 1992 al 1995), quando le inchieste giudiziarie ebbero una notevole risonanza nei media e nell'opinione pubblica, con effetti importanti nell'ambito nazionale a livello politico, sociale e culturale ${ }^{8}$.

\section{IL SISTEMA DEL DIRITTO E IL SISTEMA POLITICO}

Nella teoria dei sistemi, il diritto, inteso come sottosistema della società, ha la funzione di fornire aspettative normative generalizzate e di mantenerle stabili nel corso del tempo. Si tratta cioè di aspettative che devono rimanere valide anche quando vengano violate o deluse. Lo scopo del diritto non è dunque quello di impedire che avvengano fatti o fenomeni di natura illecita, ma quello di indicare cosa fare quando essi si verificano. In pratica il diritto deve garantire la possibilità di agire in una forma prevista una volta che una norma venga disattesa: ad esempio che venga applicata una pena di qualche tipo. In questo senso il diritto funziona come "sistema immunitario" della società", consentendo di reagire a situazioni impreviste o indesiderate.

Il sistema del diritto procede in base al codice diritto/non-diritto (o anche ragione/torto) $(\text { Recht/Unrecht })^{10}$ : vi è una comunicazione di natura giuridica ogni volta che vengono rivendicati diritti e si deve decidere, in base alle norme vigenti, chi ha ragione e chi ha torto. Il sistema si occupa di quella che Luhmann chiama la dimensione temporale del senso: il diritto cioè non garantisce l'integrazione sociale né provvede al controllo dei comportamenti - in quanto non si può impedire che la legge venga violata - ma è un mezzo per cercare di "controllare" - nel senso esposto in precedenza - il futuro (che è di per sé incerto). Norme e procedure costituiscono i cosiddetti programmi del sistema, che consentono al codice di operare: attraverso tali programmi, il diritto stabilisce (o quantomeno assegna) la ragione e il torto. I programmi del diritto non sono "di scopo" ("finalizzati a") ma "condizionali": "se..." accade una certa cosa, "allora" il diritto stabilisce cosa avverrà in seguito.

Un aspetto fondamentale consiste nel fatto che il diritto moderno è un diritto positivo, autofondato: non c'è un riferimento esterno (come la natura o Dio) ${ }^{11}$, non c'è una norma essenziale sulla quale si reggano tutte le altre (anche se ovviamente ogni ordinamento ha dei principi fondamentali). Il diritto inoltre non è "giusto": non ci possono essere soltanto norme giuste in generale, sempre e comunque, che tutti condividono. Il diritto, a differenza della politica (e dell'opinione pubblica $^{12}$ ), non si fonda sul consenso (del "popolo", dei cittadini, degli elettori, ecc.) e per questo, al contrario della politica, non necessita di riconferme periodiche. La politica infatti ha bisogno del consenso per esercitare un potere, che proprio per questo viene riconosciuto come legittimo. Tale potere viene usato per realizzare la funzione di questo sistema, che è quella di decidere in maniera vincolante per tutti: proprio grazie al potere si accettano le decisioni ${ }^{13}$. Il sistema della politica 
crea le leggi e si occupa dell'amministrazione (distinzione politica/amministrazione). Il pubblico dei cittadini rappresenta la terza componente del sistema, in quanto le organizzazioni politiche e l'amministrazione (quindi le cariche politiche e quelle amministrative) necessitano del consenso pubblico e al pubblico si indirizzano.

Il codice principale della politica è superiore/inferiore, che stabilisce chi detiene il potere. Un altro dei codici del sistema politico è governo/opposizione, che garantisce l'esercizio del potere in modo controllato e che è connesso alla democrazia: è grazie all'alternativa governo/opposizione (o anche soltanto alla possibilità di tale alternativa) che vi è democrazia, in quanto ciò significa che le posizioni sono interscambiabili. Senza opposizione, di conseguenza, non c'è democrazia.

La politica - come qualsiasi altro sistema - ha dei limiti interni ed esterni. Internamente, il sistema politico è limitato rispetto alle proprie operazioni: cioè è operativamente chiuso: funziona soltanto in base ai propri codici e programmi, ma è cognitivamente aperto ad "apprendere" dal proprio ambiente, potendo così evolvere ${ }^{14}$. Esternamente, esso è limitato da un lato - rispetto alle decisioni che può prendere (si pensi a una qualsiasi politica pubblica) - dalla disponibilità di denaro, in base a cui si stabilisce quali politiche siano possibili e quali no; dall'altro, dal diritto, in quanto la politica può fare solo ciò che è legale (che è in accordo alla legge, che è previsto e legittimato dal diritto).

\section{IL CONCETTO DI CORRUZIONE SISTEMICA}

L'etimologia della parola corruzione rimanda al latino corruptio-onis, derivato di corrumpěre e indica appunto qualcosa che viene corrotto da qualcos'altro, con conseguente decomposizione, disfacimento ${ }^{15}$. In questo senso corruzione indica che qualcosa si è rovinato, non è più integro. In generale, sembra possibile parlare di "corruzione sistemica" in due sensi. In un senso ampio, generale, comune, potremmo dire che la corruzione, è inteso come un accordo illecito, illegale, che esula dal diritto e che consiste in un pagamento in denaro o in una determinata prestazione (favore) per ottenere vantaggi. In questo senso, è possibile affermare che la corruzione è sostanzialmente un fenomeno di routine in ambito pubblico: un fenomeno sistematico di scambio di favori e pagamenti, in cui le parti coinvolte accettano di far parte del meccanismo (sistema) corruttivo perché "si sa che è così che funziona" (ovvero, anche se ingiusto, illegale, illecito, molte volte si tratta dell'unica maniera per ottenere qualcosa che non è possibile ottenere in maniera lecita). È dunque è unmodo di ottenere dei vantaggi e più in generale di essere inclusi in qualche tipo di cerchia per lo scambio di favori. Non è un caso che spesso i magistrati che si occupano di corruzione si sentano rispondere da corrotti e corruttori inquisiti che il pagamento di una tangente viene percepito come "la regola del gioco", un "qui pro quo" che seppur non desiderabile, risulta necessario ${ }^{16}$.

È anche possibile un'analisi della corruzione dal punto di vista della TSS. In questo senso parliamo di corruzione sistemica, che riguarda quantomeno tre sistemi: diritto, politica, economia. Si tratta dunque di una corruzione dei sistemi stessi, nel senso di una deviazione dal loro codice e dal loro funzionamento normale. Di fronte dunque a un fenomeno corruttivo, il codice di un sistema non viene più rispettato e il sistema viene diretto dal codice di un altro sistema. Ciò accade ad esempio quando all'interno del sistema politico non si agisce sulla base dei programmi e dei valori 
dei partiti, ma per interessi personali, o ancora quando accordi commerciali ed economici non vengono stabiliti in base a strategie di mercato, ma a partire da interessi particolari.

Mettendo a confronto le due prospettive, potremmo forse dire che la corruzione (nel senso comune di atto illegale, di reato penale) è anche e sempre una corruzione del funzionamento dei sistemi: rappresenta una dedifferenziazione fra diritto ed economia (scambio illecito di denaro) e, in ambito pubblico, anche del sistema politico (le cui decisioni vengono influenzate indebitamente). Il risultato è dunque, sul piano delle strutture sistemiche, dedifferenziazione e perdita di unità del sistema, con una minaccia riguardo il futuro del sistema stesso. Poiché infatti i sistemi continuano ad esistere in virtù della continuità delle loro operazioni, se tale meccanismo si blocca, viene compromessa la sopravvivenza del sistema stesso.

Allo stesso tempo, i sistemi hanno effetti anche su quello che nella teoria di Luhmann si chiama ambiente, cioè tutto quel che non fa parte del sistema ma che viene coinvolto in qualche modo da esso, o che gli "fornisce" materiali per le sue operazioni. Ad esempio all'interno dell'ambiente dei vari sistemi della società vanno inscritti anche gli esseri umani, i quali a loro volta possono essere considerati sistemi: in quanto corpi (sistemi biologici) o in quanto coscienze (sistemi psichici, che si riproducono grazie alla sequenza dei pensieri). Questo significa che un sistema "corrotto" avrà sul proprio ambiente, e quindi anche sugli esseri umani, conseguenze impreviste. Ad esempio, come già esposto (paragrafo 2), un giudice che condanna ingiustamente una persona, non perché sia sicuro che essa sia colpevole, ma perché è motivato da altri fattori (una tangente, un sentimento avverso), causa una corruzione sistemica, con una dedifferenziazione fra diritto e economia o fra diritto e morale, e "corrompe" anche l'ambiente, condizionando la vita di un essere umano in maniera significativa.

Riflettere sugli esseri umani come parte dell'ambiente è importante anche riguardo il problema della loro inclusione (e quindi anche della loro esclusione), ossia della loro partecipazione, del loro coinvolgimento, nella società. Pertanto, gli inclusi possono agire e comunicare in modo differente nei differenti sistemi: si può avere un lavoro oppure no, molto o poco denaro, essere buoni o cattivi genitori, avere un'istruzione avanzata o meno, ecc. In questo senso, la corruzione può essere vista come una maniera per venire inclusi, per entrare a far parte di un sistema e così avere accesso a possibilità e risorse altrimenti precluse. In questo modo favori, tangenti, organizzazioni criminali, relazioni di amicizia e reciprocità, o più in generale relazioni di natura illegale instaurate per avere qualche vantaggio (sia esso in denaro o meno), rappresentano modalità di inclusione ${ }^{17}$.

Legami e reti di questo tipo operano in maniera parassitaria rispetto alla società, e possono raggiungere grande stabilità e grande capacità di adattamento, come è ben evidente in Italia, anche a causa delle varie organizzazioni criminali "tradizionali", come mafia siciliana, la 'ndrangheta calabrese, la camorra campana, la Sacra corona unita pugliese.

Caratteristiche di tali reti sono la dinamicità, con la continua creazione di nuovi legami e di nuove forme di favori e vantaggi; una struttura gerarchica rigida; l'interazione faccia a faccia; la comunicazione orale. Inoltre, è necessario confermare continuamente la propria partecipazione, senza farsi condizionare troppo dall'esterno. La fiducia cioè, in una forma o nell'altra, va continuamente riconfermata, anche e soprattutto in virtù delle minacce sempre possibili: tutti coloro che sono coinvolti nel "sistema corruttivo" sono ricattabili, ad esempio possono essere denunciati ed 
essere arrestati - anche per questo desiderano continuare a far parte del "sistema". Si trattadi strutture forti proprio perché non si lasciano istituzionalizzare: questo le protegge anche dai tentativi di influenza esterna. Inoltre, anche se non legittime, esse possono guadagnare un certo consenso pubblico - proprio perché forniscono vantaggi o servizi che lo stato non è in grado di assicurare indebolendo le istituzioni ufficiali.

Tornando alla corruzione sistemica in senso stretto, Luhmann ha scritto che essarappresenta un problema morale della società-mondo contemporanea, in quanto il sabotaggio del codice del sistema risulta problematico anche a livello morale ${ }^{18}$. Si pensi ad esempio alla criminalità, ma anche al doping nello sport, al "commercio dell'amore", al falso con i dati della ricerca empirica. Quindi agire in base a parametri morali (cioè distinzioni come bene/male, etico/non etico, ecc.) serve non soltanto a "essere giusti", a fare il bene e non il male, ma protegge anche l'autonomia dei sistemi, tutelando di conseguenza le loro "regole", comprese quelle di inclusione degli individui.

In questo senso i media - e in particolare il giornalismo professionale -, che sono portati a trattare nei loro servizi i fatti nella forma di novità (scandali) ${ }^{19}$, incoraggiano a fare valutazioni morali. Purtroppo suscitare indignazione è facile, mentre fornire consigli o soluzioni pratiche è difficile. Per questo l'intervento del diritto è fondamentale per limitare i danni - a patto che ciò avvenga ovviamente senza il ricorso alla corruzione.

Un altro aspetto interessante dal punto di vista della teoria dei sistemi riguarda la distinzione fra struttura e semantica. La struttura è l'insieme delle relazioni fra gli elementi di un sistema. La semantica è l'insieme dei temi di rilevanza comunicativa della società ${ }^{20}$. Inoltre, struttura e semantica stanno fra loro in rapporto circolare: la semantica descrive la società, ma questa stessa descrizione a sua volta contribuisce a determinare l'orientamento dei sistemi, quindi le strutture. Soprattutto, i mutamenti strutturali possono essere colti solo a livello semantico: è importante dunque che la semantica utilizzata sia adeguata, per poter descrivere quel che avviene nella maniera più esatta.

In questo senso, la corruzione indebolisce, corrompe la struttura dei sistemi e della società nel suo complesso. Ma la corruzione è anche un tema della comunicazione: se ne parla in ambito penale, nel senso comune, si discute riguardo le sue caratteristiche e le sue forme, e riguardo le sue conseguenze. Perciò è importante analizzare come la corruzione viene concepita nella società, come viene rappresentata e come tale rappresentazione influenzi l'orientamento stesso dei vari sistemi nei confronti della corruzione. A questo proposito occorre tener conto della "percezione della corruzione", cioè di quanto i cittadini credono che la corruzione determini la loro quotidianità21. Laddove tale livello risulti alto - anche se ciò non dovesse trovare riscontro nella realtà (dati effettivi sui reati compiuti, condanne, ecc.) - si ha come conseguenza una sfiducia nei confronti della politica, dello Stato, della collettività in generale.

\section{TANGENTOPOLI E LE SUE RIPERCUSSIONI A LIVELLO DEL SISTEMA POLITICO E DELL'OPINIONE PUBBLICA}

L'espressione "Mani pulite"22 indica, come già detto, una serie di inchieste giudiziarie condotte in Italia negli anni '90. Dal 1992, un gruppo di magistrati di Milano - in particolare Antonio 
Di Pietro, Gherardo Colombo e Piercamillo Davigo (coordinati da Francesco Saverio Borrelli) indagarono sul cosiddetto scandalo di "Tangentopoli", cioè un sistema di finanziamento illecito dei partiti politici in tutto il paese. As investigações trabalharam descobrindo que este sistema de troca de propinas era muito comum. In cambio di tangenti in denaro da parte di imprenditori e uomini d'affari, molti politici facevano loro dei favori, assegnando illegalmente appalti pubblici o altri lavori pagati con denaro pubblico.

\footnotetext{
"Nella capitale morale ogni appalto doveva sovvenzionare la politica in quote prestabilite (tot alla Dc, tot al Psi, tot al Pci, ecc., secondo il consenso acquisito) e le imprese a loro volta potevano prestabilire i vincitori delle gare in barba al libero mercato, formando così un «cartello» che escludeva altra concorrenza e falsava i costi. [...] Il sistema era talmente oliato da rendere praticamente impossibile comprendere chi, tra imprese e partiti, avesse il coltello dalla parte del manico. Gli imprenditori si definiranno ricattati dai politici, i politici come assediati da imprenditori ansiosi di offrire" ${ }^{23}$
}

"Gli imprenditori, di norma, dichiarano di aver subito pressioni irresistibili e di essere stati costretti dai politici a pagare per non essere esclusi dal giro degli appalti. I politici ribattono di essere stati assediati dagli imprenditori. «Ma quale concussione, dottore dice un giorno un politico a Davigo - i concussi siamo noi: gli imprenditori ci corrono dietro per pagarci le tangenti prima che arrivino i loro concorrenti.

Stabilire le reali responsabilità dei diversi protagonisti è importante per formulare le imputazioni: corruzione (l'imprenditore paga spontaneamente per ottenere un favore dal pubblico ufficiale) o concussione (il pubblico ufficiale estorce denaro all'imprenditore minacciando di tagliarlo fuori)?"24

Questo sistema era tanto comune da non essere di fatto percepito come irregolare: come abbiamo visto nel paragrafo precedente, pagare una tangente viene considerato tutto sommato necessario dalle parti in gioco per continuare a lavorare, per sopravvivere all'interno di un sistema (intendendo qui il termine in un senso ampio e generale) dal funzionamento consolidato.

Mani pulite - e cioè l'insieme delle indagini giudiziarie e il loro "racconto" da parte dei media - viene considerata uno dei fattori che hanno causato o quantomeno accompagnato il passaggio dalla cosiddetta "Prima" alla "Seconda Repubblica". In quegli anni (1992-1994) infatti vi furono vari mutamenti nel panorama politico-istituzionale italiano, in buona parte proprio in conseguenza di Mani pulite. Ad esempio, cambiò il sistema elettorale, vennero creati nuovi partiti politici mentre altri si sciolsero. Indagini e processi, dunque - ossia il sistema del diritto - contribuirono a questo cambiamento, sia perché coinvolsero molte persone che rivestivano importanti incarichi politici(e che di conseguenza smisero di fare politica), sia perché spinsero tutta la classe politica a ripensare se stessa di fronte alle richieste di legalità che venivano dall'opinione pubblica, sdegnata da quanto stava accadendo.

I media contribuirono a creare un tale clima, raccontando in maniera dettagliata e a volte ossessiva l'inchiesta, tanto che molti giornalisti manifestavano apertamente il loro appoggio ai giudici. ${ }^{25}$ Ciò ovviamente cozza con l'etica professionale, che imporrebbe ai giornalisti di restare equidistanti dai fatti raccontati e dalle persone in essi coinvolte.

Nel linguaggio della teoria dei sistemi, potremmo dunque dire che Mani pulite creò i presupposti per un mutamento dell'assetto politico-istituzionale italiano: l'inchiesta e i processi che 
seguirono provocarono delle "irritazioni" nei confronti degli altri sistemi, in particolare di quello politico. Detto altrimenti: crearono delle instabilità e dei condizionamenti, che costituirono un impulso al cambiamento.

\section{LE CONSEGUENZA DI TANGENTOPOLI NELL'AMBITO DEI DIRITTI UMANI}

Crediamo sia possibile, nell'ambito dell'inchiesta Mani pulite, discutere delle conseguenze della corruzione sui diritti umani sia al livello dell'impatto delle indagini sui diritti degli indagati che a quello relativo alle conseguenze sulla vita della comunità.

In un'intervista al magistrato Gherardo Colombo ${ }^{26}$, pubblicata il 23 luglio 2018 sul quotidiano «la Repubblica», il giornalista Piero Colaprico ricorda un episodio: il magistrato che ordina alle forze dell'ordine di "guardare a vista" le persone arrestate nell'inchiesta. Colombo risponde raccontando che uno degli imputati più in vista all'epoca, Sergio Cusani, non poteva neanche andare in bagno da solo: era sempre sorvegliato da qualcuno, anzitutto per scongiurarne il suicidio, come avvenuto - e qui trova giustificazione l'episodio richiamato dal giornalista - nel caso dell'imprenditore Raul Gardini, che si era tolto la vita poche ore prima di essere interrogato. Ciò aveva indotto i magistrati a disporre misure di controllo che in una situazione ordinaria non sono giustificabili e che ledono diritti essenziali dell'individuo.

In questo senso, ad esempio, è stato calcolato che i suicidi legati in qualche modo alle inchieste di Mani pulite sono stati 11 nel 1992, 10 nel 1993 e 10 nel $1994^{27}$.

Ci sono state poi molte polemiche a proposito della carcerazione preventiva, cioè quello strumento che permette di tenere gli indagati in carcere, allo scopo di evitare l'inquinamento delle prove. Secondo molti (cittadini, giornalisti, politici, ecc.) i magistrati di Mani Pulite abusarono di tale strumento.

Qui non ci interessa discutere se i diritti umani di qualche indagato siano stati violati o meno. Interessante è però osservare come in nel caso di grandi inchieste giudiziarie particolarmente clamorose, alcune misure previste dal diritto stesso, come la carcerazione preventiva, abbiano conseguenze dirette sui diritti umani fondamentali: è giusto limitare la libertà personale prima di un giudizio? Con quali eccezioni? E fino a che punto? Si pensi ad esempio al caso drammatico dell'imprenditore Gabriele Cagliari, imprigionato per quattro mesi, e che si è poi suicidato, lasciando una lettera di spiegazione, in seguito resa pubblica ${ }^{28}$.

Dall'altro lato, va ricordato che attorno alle inchieste si creò un clima piuttosto esasperato, che, è facile immaginare, avrà in qualche modo condizionato l'operato all'interno del sistema giudiziario. Soprattutto però è possibile constatare che sottosistemi differenti si sono influenzati reciprocamente, contribuendo alla creazione di tale clima: i media che raccontavano le inchieste concentrandosi sui protagonisti - positivi (magistrati) e negativi (politici e imprenditori indagati o presunti corrotti) - hanno condizionato l'opinione pubblica ma anche la politica e la magistratura. Inoltre nel dibattito pubblico hanno preso vita delle fazioni: si è parlato molto ad esempio della contrapposizione fra giustizialisti e garantisti. I primi a "fare il tifo" per i magistrati, reclamando processi rapidi, con pene non soltanto giuste ma anche e esemplari; i secondi a ricordare i diritti di 
indagati e imputati, in particolare la presunzione di innocenza, sostenendo che compito del diritto (ovvero del sistema giudiziario) è di concentrarsi sulle responsabilità personali, senza lasciarsi condizionare da altri fattori e da influenze varie provenienti dalla politica o dalla società in senso lato. Si potrebbe così intendere il giustizialismo come una forma di "violenza", anche in senso sistemico: una pressione esercitata dall'opinione pubblica nei confronti del sistema giudiziario; una pressione tuttavia indebita, poiché diretta a negare la presunzione di innocenza. Un diritto che può essere o meno considerato un diritto umano, ma la cui negazione costituisce senza dubbio una fra le violazioni peggiori che si possano subire, in quanto priva l'individuo della libertà.

Il secondo aspetto dell'analisi relativa al rapporto tra corruzione e diritti umani nell'inchie- sta Mani pulite riguarda le conseguenze della prima sulla vita sociale più generale. È evidente che i fenomeni corruttivi negano la regolarità delle decisioni in determinati ambiti: politica, economia, giustizia, ecc. Di conseguenza, in tali ambiti, tutti gli attori (individuali e collettivi, privati e pubblici) che risultano coinvolti da tali decisioni subiscono, consapevolmente o inconsapevolmente, delle ricadute. Le decisioni irregolari, in altre parole, condizionano la vita di individui, associazioni, imprese, apparati dello Stato, e via dicendo. Un esempio banale: una tangente può determinare il destino di un'opera pubblica di grande importanza (un ospedale, un'autostrada, ecc.): ciò influenzerà la vita dei cittadini e della comunità. In questo senso, valutare con precisione (a livello quantitativo e qualitativo) le conseguenze della corruzione sui diritti umani risulta difficile.Un modo per ridurre la complessità dell'analisi potrebbe essere l'analisi empirica ${ }^{29}$. Tuttavia, anche soltanto la "percezione della corruzione", come detto, risulta importante: il fatto di credere che la corruzione in un determinato paese sia elevata, condiziona la vita dei cittadini, che si sentono privati di un diritto fondamentale: quello di poter agire senza condizionamenti occulti nella realtà sociale. La legalità delle decisioni è infatti un presupposto essenziale per l'effettivizzazione di tutti i diritti individuali e collettivi.

\section{CORRUZIONE E "VIOLENZA SISTEMICA"}

Qual è dunque lo scopo che giustifica l'uso dell'espressione “violenza sistemica”? E soprattutto: quale utilità può avere un tale sforzo?

"Corruzione" nel senso comune e "corruzione sistemica" sono due cose diverse, come abbiamo visto: la prima è una situazione giuridicamente (e moralmente) impropria, irregolare. La seconda è una situazione che mette in pericolo i sistemi e, di conseguenza, la loro funzione nella società. La dedifferenziazione, allora - prima ancora che un problema morale - rappresenta una minaccia nei confronti della struttura della società e della sua sopravvivenza. E poiché il funzionamento dei sistemi ha conseguenze sul loro ambiente, e di questo fanno parte anche gli esseri umani, gli effetti su questi ultimi possono essere importanti e seri, con sofferenze e limitazioni ingiuste e difficili da sopportare.

Oltre a ciò, immaginare situazioni e contesti di dedifferenziazione significa immaginare come la società potrebbe cambiare e quali problemi potrebbero presentarsi in proposito. Ad esempio, se gli apparati giudiziario-investigativi agiscono sotto l'influenza della politica o dei media, non si potrà sicuramente realizzare una giustizia non soltanto "giusta" (come in fondo tutti vorremmo, anche 
se il diritto in quanto sistema sociale non è, né può essere, giusto), ma neanche equa o omogenea. $\mathrm{O}$ se i media - che fondano i loro racconti sul criterio della novità, lasciando in secondo piano qualsiasi altro fattore - tendono a selezionare ed enfatizzare determinati fatti con lo scopo di ottenere maggior seguito o di offrire sostegno a personaggi o organizzazioni politiche, essi spettacolizzano le vicende raccontate e contribuiscono a creare un clima di esasperazione nell'opinione pubblica. Di conseguenza - al di là del fatto di constatare un giornalismo di scarsa qualità e poco affidabile - si avranno pesanti conseguenze sul diritto all'informazione e, cosa ancor più preoccupante, un deficit di controllo del potere.

Tutto ciò conduce a un problema relativo alla fiducia e al futuro. Vi è difatti un collegamento tra questi due elementi: la fiducia serve a vincolare il futuro e a ridurre la complessità sociale ${ }^{30}$. Così, in un mondo sempre più complesso, più difficile da capire, diminuisce il senso di familiarità che ognuno percepisce rispetto ai fatti sociali, e di conseguenza, per colmare tale carenza, si ha un crescente bisogno di far ricorso alla fiducia. Da una parte, la fiducia dei cittadini nei confronti delle istituzioni, della politica, ma anche dei media stessi, risulta indebolita, non soltanto a causa della corruzione stessa, ma anche dalla sua rappresentazione (e relativa percezione). L'esasperazione del dibattito pubblico conduce a una polarizzazione delle posizioni, a una generalizzazione dei giudizi, alla cosiddetta "anti-politica".

Di conseguenza il futuro diventa incerto: senza fiducia la politica in particolare soffre, perché in mancanza di un solido sostegno popolare risulta difficile avere governi duraturi che possano realizzare riforme importanti. Allo stesso tempo, in un contesto viziato dalla corruzione, il futuro diventa un azzardo proprio perché le decisioni influenzate dalla corruzione ostacolano il sistema politico e il funzionamento degli altri sistemi, generando situazioni impreviste che avranno a loro volta ulteriori conseguenze impreviste e imprevedibili.

\section{CONCLUSIONI. CORRUZIONE E PERCEZIONE DELLA CORRUZIONE: AUTO-OSSERVAZIONE?}

Se la corruzione in generale ma anche la corruzione in senso sistemico implicano e rappresentano forme di violenza, è anche vero che esse indeboliscono o rendono impossibile la fiducia, base della solidarietà sociale. Come abbiamo visto, già soltanto la percezione della corruzione causa tale indebolimento nei sottosistemi della società ma anche nelle organizzazioni e nelle interazioni (ulteriori tipologie di sistemi sociali). Prendiamo ad esempio in considerazione il sistema scientifico, e nello specifico le scienze sociali: la loro funzione (stabilire la verità, cioè quel che è scientificamente comprovato) in questo caso si declina nell'identificare le situazioni in cui la percezione della corruzione corrisponde e quelle in cui non corrisponde alla realtà documentata e nel valutare l'impatto stesso di tale percezione nel mondo sociale (calcolando anche i "danni" provocati da una percezione "errata" del fenomeno: ad es. inasprimento del dibattito pubblico, paura nei cittadini, ecc.).

Il problema è che molte ricerche sulla corruzione si concentrano o si limitano alla percezione della corruzione stessa: si veda ad esempio l'indice prodotto da Transparency International a partire dal $1995^{31}$ o il Global Corruption Barometer, realizzato dal $2003^{32}$. 
In questo senso, ad esempio, l'Italia è uno dei paesi in cui la percezione della corruzione è più elevata $^{33}$. Tuttavia, questo potrebbe semplicemente significare che l'intolleranza della popolazione è molto elevata: essendo cioè la corruzione percepita - per lo meno al livello del senso comune

- come inaccettabile, sarebbe minore la "soglia di tolleranza" dei cittadini e della collettività in generale ${ }^{34}$. Saremmo dunque di fronte al cosiddetto paradosso di Trocadero: "Più si perseguono i fenomeni corruttivi sul piano della prevenzione e le fattispecie di reato sul piano della repressione, maggiore è la percezione del fenomeno" ${ }^{35}$.

Come si potrebbe dunque cercare di distinguere la percezione e la corruzione? Qualche suggerimento lo dà il sociologo italiano Piergiorgio Corbetta ${ }^{36}$ : citando un rapporto (Eurobarometro) dell'Unione europea sulla corruzione nel 201737, egli distingue: 1) l'accettabilità della corruzione (grado di tolleranza del fenomeno): in questo l'Italia è al di sotto della media europea, cioè la corruzione è meno tollerata rispetto alla media; 2) l'esperienza diretta della corruzione (il fatto di conoscere - o meno - qualcuno che è stato corrotto o che ha corrotto): a questo livello l'Italia è nella media europea; 3) la percezione stessa della corruzione: in questo senso "l'Italia è drammaticamente sopra alla media europea e fra i Paesi che si auto-ritengono più corrotti" ${ }_{38}$. Corbetta cita inoltre anche un'indagine dell'Istituto nazionale di statistica italiano ${ }^{39}$ secondo cui "negli ultimi 12 mesi» solo l'1,2\% degli intervistati ha dichiarato di essere stato coinvolto direttamente in eventi corruttivi quali richieste di denaro, favori, regali o altro in cambio di servizi dovuti" 40 .

Tali riflessioni dunque suggeriscono qualcosa che, per la TSS, rappresenta un leit-motiv: è necessario descrivere in maniera migliore, distinguendo, ovvero attraverso distinzioni (criteri, schemi, categorie, strumenti intellettuali) più raffinate e scientifiche, alla condizione di restare sempre consapevoli che la scienza, nel momento in cui descrive, descrive anche se stessa, il suo modo di lavorare, di osservare. In questo senso, la società descrive se stessa attraverso la sociologia, cioè una forma di rappresentazione della società che è allo stesso tempo un prodotto della società ${ }^{41}$.

Pertanto, potremmo comprendere la percezione della corruzione come una forma, un mezzo per descrivere la società: se, nel senso comune, tale semantica è influenzata da fattori non scientifici (di tipo socioculturale, come l'influenza dei media, ecc.), il ruolo della scienza è quello di realizzare una descrizione più approfondita. Tuttavia, è importante che le sue descrizioni siano "comprensibili" anche dai media, in modo che una comprensione dei fatti più "autentica" possa diffondersi al di fuori della cerchia degli intellettuali.

\section{NOTAS}

1 Una prima versione di questo articolo è stata presentata nel giugno del 2019 durante il XXVIII Encontro Nacional do Conpedi (Conselho Nacional de Pesquisa e Pós-graduação em Direito) di Goiânia. Esso è frutto di una ricerca iniziata nel 2018 e tuttora in corso. Finanziata dalla CAPES, si intitola: "OBSERVAÇÃO SISTÊMICA DA VIOLÊNCIA COMO SISTEMA ORGANIZACIONAL NA CRIMINALIDADE EM REDE E SEUS REFLEXOS NO MERCADO: ANÁLISE COMPARATIVA ENTRE A OPERAÇÃO MÃOS LIMPAS E A OPERAÇÃO LAVA JATO". Il lavoro vede la collaborazione delle seguenti università: Universidade Federal do Rio Grande do Sul (UFRGS), Universidade do Vale do Rio dos Sinos (UNISINOS), Centro Universitário Ritter dos Reis (UniRitter) Laureate International, Unijuí, Universidade Presbiteriana Mackenzie, Universidade Federal do Sergipe, Università Roma Tre, Università degli Studi di 
Salerno, Università degli Studi di Chieti e Pescara. L'obiettivo della ricerca è quello di rilevare le conseguenze della corruzione a livello delle politiche pubbliche e i suoi riflessioni sui cittadini, identificando le nuove forme di "violenza sistemica" a partire da due eventi: 1" "operazione Lava Jato" in Brasile e il complesso di indagini denominato "Mani Pulite", che hanno permesso di identificare il cosidetto sistema criminale noto come "Tangentopoli", in Italia. Alcuni dei contenuti di questo articolo sono stati presentati nel 2018 nel corso di due eventi: il I Seminário Internacional "Violência sistêmica e seus impactos nos direitos humanos" svoltosi il 31 agosto presso la UniRitter (Porto Alegre), e il I Seminário Internacional "Gestão do Estado e Políticas Públicas: desafios para o controle da corrupção" del 13 settembre presso la Universidade Presbiteriana Mackenzie (São Paulo).

2 Da qui in avanti: TSS.

3 Un aspetto che non è qui possibile approfondire riguarda il tipo di società che ci si pone di realizzare. Di fatto, è impossibile pensare a qualsiasi forma concreta di giustizia e democrazia se prima non si identificano i modelli relativi all'equilibrio fra poteri e al ruolo dell "individuo all” interno della realtà sociale. A questo proposito, si può parlare, ad esempio, di due modelli classici: quella di una società verticale (storicamente rinvenibile in differenti contesti) e quello di una società orizzontale (ideale). Nel primo, gerarchicamente determinato, "la persona non è un fine, non va salvaguardata, può trasformarsi in strumento per la promozione dei più validi rappresentanti della specie destinati a loro volta a promuoverla ulteriormente e può (sarebbe da dire "deve") essere eliminata quando non serve o reca danno. Ne deriva che la giustizia consiste nel promuovere e tutelare le gerarchie; nel dare dignità ai privilegi; nell'eliminare, anche fisicamente, chi è dannoso": COLOMBO, 2010, p. 43. Nel secondo modello, "qualunque persona, per il fatto stesso di esistere, costituisce uno scopo, un fine, una dignità da salvaguardare, e la segregazione diventa un controsenso, ammissibile soltanto in casi rari ed eccezionali" (50). Di conseguenza, "Il modello orizzontale non tollera alcuna opacità delle istituzioni: l'amministrazione non può non essere trasparente" (55), in quanto "Il mescolamento delle due concezioni della società e dei due modelli di organizzazione determina una gamma quasi infinita di diverse possibilità, il tipo di disciplina del vivere comune, e di conseguenza il senso della parola "giustizia"." (58).

4 Colombo (2010), per descrivere in maniera completa e soddisfacente la complessità del tema (corruzione) e fino a che punto il fenomeno si verifichi nell'Italia contemporanea, inaugura il suo testo con una descrizione, verosimile, di un paese immaginario in cui le pratiche corruttive avvengano trasversalmente, in tutti gli ambiti. Gli esempi sono molti: dalla sfera pubblica a quella privata, dall'ambito sanitario alla banche, dall'ambito amministrativo alla giustizia, dal giornalismo all'artigiano, ecc.

5 È possibile pensare, in primo luogo, alla teoria critica della Scuola di Francoforte e alla tradizione ad essa seguente, in particolare ad Habermas.

6 Si pensi, in particolare, all'economia: dove sta l'utilità nel dire che una operazione (economica) non è "buona", ossia moralmente accettabile? Il modo in cui funziona l'economia non può essere messo in questione: differenti possono soltanto essere le decisioni prese dai singoli, ma non il fatto che quando si decida, in ambito economico, lo si faccia attraverso la distinzione avere/non avere o pagare/non pagare.

7 Occorre qui intendersi sul significato dell'espressione "funzionamento della giustizia": "Quando si dice che la giustizia non funziona, si allude alla sua amministrazione; invece quando si dice che in un paese non c'è giustizia, ci si riferisce al principio fondamentale della convivenza. Si dice anche che le leggi (e/o i comportamenti) di un paese non sono conformi alla giustizia.": COLOMBO, 2010, p. 24. In questo senso, è fondamentale considerare il rapporto fra giustizia e regole (norme): "La giustizia non può funzionare se i cittadini non comprendono il perché delle regole. Se non lo comprendono tendono a eludere le norme, quando le vedono faticose, e a violarle, quando non rispondono alla loro volontà.": COLOMBO, 2010, p. 8 (corsivo nell'originale). 
8 "Nel linguaggio giornalistico, città in cui è diffuso il malcostume di pretendere e incassare tangenti, ossia somme di denaro richieste in cambio di favori, concessioni o altre forme d'intermediazione illecite da parte di chi è in grado d'influenzare la buona riuscita di tali affari o pratiche. Per estensione, il fenomeno, lo scandalo delle tangenti nella pubblica amministrazione e in ambienti politici. L'uso del termine si è affermato a partire dal 1992 in seguito alle inchieste giudiziarie svolte dalla magistratura di Milano e successivamente condotte anche in altre città d'Italia, che portarono alla dissoluzione di alcuni partiti storici italiani (tra cui DC e PSI)": ENCICLOPEDIA ONLINE TRECCANI.

9 LUHMANN, 1990, p. 580.

10 Sul sistema del diritto, si veda in particolare LUHMANN, 1993.

11 “All'inizio della storia dell'umanità [...] Diritto e giustizia allora coincidevano, perché si riteneva che il primo provenisse direttamente da dio. [...] il diritto era un'emanazione di dio, e le leggi continuavano a essere giuste per definizione.”: COLOMBO, 2010, p. 27.

12 Non è per caso che la politica deve fare riferimento all'opinione pubblica ed esortare i cittadini ad assumersi la quelle responsabilità che gli stessi cittadini, exortando eles ao compromisso em nome dos mesmos valores - come chiarezza, coerenza, impegno e partecipazione - esigono dalla politica: si veda COLOMBO, 2010, p. 146-147.

13 Ovviamente si tratta sempre di un potere che non è assoluto: la società non dipende totalmente da un potere centrale autoritario.

14 Si parla qui di evoluzione intesa non in quanto sinonimo di "progresso", "miglioramento", ma come mutamento e complessità crescente: cfr., fra gli altri, LUHMANN, 2009 e 1997.

15 DIZIONARIO ONLINE TRECCANI: http://www.treccani.it/vocabolario/corruzione/.

16 Per un resoconto complessivo delle indagini e delle azioni dei magistrati di Milano e una analisi generale di Mani Pulite/Tangentopoli, cfr., fra gli altri, BARBACETTO; GOMEZ; TRAVAGLIO, 2017; FACCI, 2010.

17 Su questo, si veda in particolare LUHMANN, 2005, pp. 226-251.

18 Cfr. LUHMANN, 1997.

19 Sulla "realtà dei mass-media", cfr. LUHMANN, 1996.

$20 \mathrm{Su}$ questo, si veda LUHMANN, 1980. $21 \mathrm{Si}$ vedano le sezioni 7 e 8.

22 Sull'origine di questa espressione, si veda IARICCI, 2014, p. 161 (in nota).

23 FACCI, 2010, p. 101.

24 BARBACETTO; GOMEZ; TRAVAGLIO, 2017.

25 Un esempio: "Nacque, per un limitato ma decisivo periodo, una specie di Pool dei giornalisti: una redazione giudiziaria unificata con distribuzione equanime delle notizie e dei celebri verbali, spesso tradotti dal burocratese e semplificati in linguaggio corrente. Due gli obiettivi: gestire la mole impressionante delle notizie e proteggersi da eventuali censure distribuendo le notizie a tutti gli altri. Di fatto, l'informazione si fece unificata da giornale a giornale.”: FACCI, 2010, p. 121.

26 COLOMBO, 2018.

27 BUFFA, 2017.

28 Di seguito qualche passo della lettera: "L'obiettivo di questi magistrati, quelli della Procura di Milano in modo particolare, è quello di costringere ciascuno di noi a rompere, definitivamente e irrevocabilmente, con quello che loro chiamano il nostro "ambiente". Ciascuno di noi, già compromesso nella propria dignità agli occhi dell'opinione pubblica per il solo fatto di essere inquisito o, peggio, essere stato arrestato, deve adottare un atteggiamento di "collaborazione" che consiste in tradimenti e delazioni che lo rendano 
infido, inattendibile, inaffidabile: che diventi cioè quello che loro stessi chiamano un "infame". [...] I magistrati considerano il carcere nient'altro che uno strumento di lavoro, di tortura psicologica, dove le pratiche possono venire a maturazione, o ammuffire, indifferentemente, anche se si tratta della pelle della gente.": CAGLIARI, 1992. Rispondendo alle critiche nei confronti dei magistrati di Mani Pulite, rispetto alle loro presunte responsabilità in questi suicidi, si veda ad esempio una dichiarazione del magistrato Gerardo D'Ambrosio: "Noi ci siamo limitati a scoprire e perseguire fatti previsti dalla legge come reati. Poi c'è ancora qualcuno che si vergogna e si suicida": BARBACETTO; GOMEZ; TRAVAGLIO, 2017, p. 108. Ad ogni modo è facile credere che proprio la carcerazione preventiva motivasse a confessare: "Era il carcere, irrogato o temuto, che stimolava le collaborazioni. [...] Confessavano perché erano in galera e volevano uscire, cioè. Confessavano perché non volevano finirci, bene che andasse. [...] La prassi di Mani pulite, fin dall'inizio, aveva ipotizzati reati i più gravi possibile così da giustificare ogni volta la carcerazione preventiva [...] quanti dei 1254 condannati di Mani pulite abbiano subito delle carcerazioni preventive a dispetto di pene poi risultate inferiori ai 2 anni, ossia condanne a cosiddette «pene sospese», con la condizionale: quasi tutti. [...] chi «parlava» poteva uscire e quindi evitare la gogna pubblica e la rovina economica.": FACCI, 2010, pp. 294-295. Riguardo alle tecniche utilizzate dai magistrati per convincere gli indagati a raccontare le loro responsabilità, si prenda ad esempio questo passo: "Il gip Italo Ghitti disse chiaro e tondo: «Il nostro obiettivo è colpire un sistema, non le singole persone». Cominciò una nuova fase giurisprudenziale. Ogni reato ipotizzato sarebbe stato inquadrato nell'affiliazione a un sistema, e dimostrare che l'indagato ne avesse fatto parte sarebbe bastato a giustificare il protrarsi delle carcerazioni. Chi parlava e denunciava altri, invece, poteva essere liberato perché ritenuto ormai inaffidabile agli occhi del sistema: come i pentiti con la mafia. Ogni proposta di sistematizzare la confessione sarebbe stata avallata dal Pool. E ogni tentativo di limitare le carcerazioni sarebbe stato chiamato «colpo di spugna».": FACCI, 2010, p. 112.

29 Nonostante sia stato finora realizzato un numero ingente di congressi, tesi di laurea e dottorato sul tema della corruzione, le ricerche di stampo empirico sono ancora poche.

30 'Dove c'è la fiducia ci sono più possibilità di esperienza e di azione, e aumentano sia la complessità del sistema sociale sia il numero di possibilità che esso può conciliare con la sua struttura, poiché con la fiducia abbiamo a disposizione una più efficace forma di riduzione della complessità": LUHMANN, 2002, p. 11.

31 TRANSPARENCY INTERNATIONAL. Corruption Perception Index. Url: https://www.transparency. org/research/cpi/overview.

32 TRANSPARENCY INTERNATIONAL. Global Corruption Barometer. Url: https://www.transparency. org/research/gcb/overview.

33 'Sull'entità del fenomeno della corruzione nel nostro Paese molti hanno scritto, commentato, dibattuto e polemizzato. Alcuni addirittura sono giunti a fornire grandezze economiche alla misura del malaffare, indicando cifre più che allarmanti, impossibili e indimostrabili, sul piano scientifico. Questi arditi esperimenti, prodotti nei laboratori di Enti, Istituzioni e centri di ricerca e ripresi a piene mani dal sistema della comunicazione, al di là della loro appropriatezza e attendibilità, hanno comunque contribuito all'edificazione $\mathrm{o}$, quantomeno, al rafforzamento dell'immagine dell'Italia come Paese corrotto, anzi, tra i più corrotti in assoluto.": FARA; TARTAGLIA POLCINI, 2019 (neretto nell'originale). "L'Italia, in ambito OCSE, è il Paese con la più alta corruzione percepita (circa $90 \%$ ) e con una fiducia nel Governo superiore al $30 \%$, più alta di quella di Grecia, Portogallo, Spagna e Slovenia nonostante questi paesi abbiano una percezione della corruzione inferiore a quella italiana (tra 1 $80 \%$ e il 90\%). | Qualcuno definisce questo fenomeno "sindrome del Botswana", inteso come tendenza ad accostare il nostro Paese a Stati difficilmente assimilabili all'Italia per livello di benessere e di ricchezza.": EURISPES, 2019.

34 Come esempi del dibattito in corso sull'utitlità dei ranking della corruzione, si veda: CANTONE;

CARLONI, 2019; FACCI, 2019. 
35 TARTAGLIA POLCINI, 2017. Si veda anche. EURISPES, 2019.

36 CORBETTA, 2017.

37 EUROPEAN UNION, 2017.

38 CORBETTA, 2017.

39 ISTAT, 2017.

40 CORBETTA, 2017 (neretto nell'originale).

41 Cfr. in particolare LUHMANN, 1997.

\section{BIBLIOGRAFIA}

BARBACETTO, Gianni; GOMEZ, Peter; TRAVAGLIO, Marco. Mani Pulite. 25 anni dopo. PaperFirst, 2017.

BUFFA, Dimitri. Suicidi o rovinati, l'eredità di Mani Pulite. Il Tempo, 16 fev. 2017.

CAGLIARI, Gabriele. 1992: questa è la realtà, non la fiction. La lettera del suicida Gabriele Cagliari. Tempi, 27 mar. 2015. Disponível em: https://www.tempi.it/1992-realta-non-fiction-lettera-suicida-gabriele-cagliari.

CANTONE, Raffaele; CARLONI, Enrico. Perché parlare di corruzione. la Repubblica, 19 jan. 2019, pp. 4-5.

COLOMBO, G. Sulle regole. Milano: Feltrinelli, 2010.

"La fine di Gardini aprì gli occhi di tutti sul sistema tangenti”, intervista di P. Colaprico, «la Repubblica», 23 luglio 2018, pp. 18.

CORBETTA, Piergiorgio. Siamo un Paese corrotto?. Rivista il Mulino, 18 dez. 2017. Disponível em: https:// www.rivistailmulino.it/news/newsitem/index/Item/News:NEWS ITEM:4199.

DIZIONARIO ONLINE TRECCANI. Corruzione. Disponível em: http://www.treccani.it/vocabolario/corruzione/.

ENCICLOPEDIA ONLINE TRECCANI. Tangentopoli. Disponível em: http://www.treccani.it/enciclopedia/tangentopoli.

EURISPES. La corruzione tra realtà e rappresentazione. Ovvero come si può alterare la reputazione di un paese. eurispes.eu, 10 jan. 2019. Disponível em: http://www.eurispes.eu/eurispes-comunicato-ricerca-corruzione-tra-realt $\% \mathrm{C} 3 \% \mathrm{~A} 0$-e-rappresentazione.

EUROPEAN UNION. Special Eurobarometer 470: Corruption. 2017. Disponível em: https://data.europa. eu/euodp/data/dataset/S2176_88_2 470_ENG.

FACCI, Filippo. Di Pietro. La storia vera. Milano: Mondadori, 2010.

. La corruzione esisterà anche ma non quanto ci dicono..., Libero, 31 jan. 2019, p. 1.

FARA, Gian Maria; TARTAGLIA POLCINI, Giovanni. Corruzione: Cantone ha ragione ma l'indice va ripensato. eurispes.eu, 22 jan. 2019. Disponível em: http://www.eurispes.eu/corruzione-cantone-ha-ragione-ma-1\%E2\%80\%99indice-va-ripensato.

ISTAT. La corruzione in Italia: il punto di vista delle famiglie, 12 out. 2017. Disponível em: https://www. istat.it/it/files/2017/10/La-corruzione-in-Italia.pdf.

LUHMANN, Niklas. Gesellschaftsstruktur und Semantik. Frankfurt am Main: Suhrkamp Verlag, 1980. 
. Sistemi sociali. Fondamenti di una teoria generale. Bologna: il Mulino, 1990 (Soziale Systeme.

Grundriß einer allgemeinen Theorie. Frankfurt am Main: Suhrkamp Verlag, 1984).

Das Recht der Gesellschaft. Frankfurt am Main: Suhrkamp Verlag, 1993.

. Die Realität der Massenmedien. Opladen: Westdeutscher Verlag GmbH, 1996.

. Die Gesellschaft der Gesellschaft. Frankfurt am Main: Suhrkamp Verlag, 1997.

.Lafiducia. Bologna:ilMulino, 2002(Vetrauen.Ein Mechanismusder Reduktionsozialer Komplexität.

Stuttgart: Lucius \& Lucius, 2000, IV ed. (1968)).

Inklusion und Exklusion. In: Soziologische Aufklärung. Vol. 6, Wiesbaden: VS Verlag für Sozialwissenschaften, 2005 (1995), pp. 226-251 (Inclusão e exclusão. In: DUTRA, Roberto; BACHUR, Jõao Paulo (Orgs.). Dossiê Niklas Luhmann. Belo Horizonte: UFMG, 2013).

Einführung in die Theorie der Gesellschaft. BAECKER, D. (Org.). Heidelberg: Carl-Auer-Systeme Verlag, 2009.

TARTAGLIA POLCINI. Giovanni. Il Paradosso di Trocadero. Il diritto penale della globalizzazione. 22 ottobre 2017; Disponível em: http://www.dirittopenaleglobalizzazione.it/il-paradosso-di-trocadero/.

TRANSPARENCY INTERNATIONAL. Corruption Perception Index. Disponível em: https://www.transparency.org/research/cpi/overview.

. Global Corruption Barometer. Disponível em: https:/www.transparency.org/research/gcb/overview.

Data de submissão do artigo: Agosto de 2019

Data de aceite do artigo: Novembro de 2019 